\title{
Clinical activity of fulvestrant in metastatic breast cancer previously treated with endocrine therapy and/or chemotherapy
}

Mi Hwa Heo, Hee Kyung Kim, Hansang Lee, Ji-Yeon Kim, Jin-Seok Ahn, Young-Hyuck Im, and Yeon Hee Park

Division of Hematology-Oncology, Department of Medicine, Samsung Medical Center, Sungkyunkwan University School of Medicine, Seoul, Korea
Received: July 4, 2017

Revised : August 9, 2017

Accepted: October 27, 2017

\section{Correspondence to}

Yeon Hee Park, M.D.

Division of HematologyOncology, Department of Medicine, Samsung Medical Center, Sungkyunkwan University School of Medicine, 81 Irwon-ro, Gangnam-gu, Seoul 06351, Korea

Tel: +82-2-3410-1780

Fax: +82-2-3410-1754

E-mail:yhparkhmo@skku.edu
Background/Aims: We conducted a retrospective analysis of the clinical activity of fulvestrant in postmenopausal women with hormone receptor-positive, human epidermal growth factor receptor 2 (HER2)-negative metastatic breast cancer (MBC) previously treated with endocrine therapy and/or chemotherapy.

Methods: We reviewed the medical records of all patients with MBC treated at Samsung Medical Center between January 2009 and August 2016. Patients received fulvestrant $250 \mathrm{mg}$ intramuscularly every 28 days (from January 2009 to November 2010) or $500 \mathrm{mg}$ intramuscularly every 28 days (from December 2010 to August 2016). Tumor responses were assessed every 8 weeks and at the end of treatment, as well as when disease progression was suspected.

Results: A total of 84 patients were included in this study. A median of two previous endocrine treatments had been performed; $79 \%$ of the patients had received two or more endocrine treatments. Forty-five patients (54\%) had been treated with chemotherapy for MBC before the fulvestrant treatment course. Visceral metastasis was found in 49 patients (58\%). The estimated median progression-free survival and overall survival were 4.4 months (95\% confidence interval [CI], 3.4 to 5.5 ) and 32.5 months ( $95 \%$ CI, 17.6 to 47.4 ), respectively. The disease control rate was $40.5 \%$ ( $95 \%$ CI, 30.5 to 51.5 ); partial response was observed in $16 \%$ of the patients and stable disease was observed in $25 \%$ of the patients. The most frequently reported adverse reactions were mild-to-moderate grade myalgia (10.5\% of the patients), injection site pain (7\%), and fatigue (7\%). Fulvestrant was generally well tolerated.

Conclusions: Fulvestrant showed encouraging clinical activity and favorable feasibility in postmenopausal women with MBC who had been treated with multiple endocrine therapies and/or cytotoxic chemotherapies.

Keywords: Fulvestrant; Salvage therapy; Breast neoplasms; Postmenopause; Female

\section{INTRODUCTION}

Aromatase inhibitors (AIs) and tamoxifen are standard therapeutic options in the first-line treatment of postmenopausal patients with hormone receptor-positive metastatic breast cancer (MBC) [1]. Despite receiving appropriate treatment, the majority of patients eventually progress or relapse during or after first-line endocrine therapy. These patients are often treated with sequential endocrine therapy. Fulvestrant is a selective estrogen re- 
Table 1. Demographic and baseline characteristics $(n=84)$

\begin{tabular}{|c|c|}
\hline Characteristic & Value \\
\hline Age, yr & $57.7(31-82)$ \\
\hline \multicolumn{2}{|l|}{ Dose of fulvestrant } \\
\hline $250 \mathrm{mg}$ & $24(29)$ \\
\hline $500 \mathrm{mg}$ & $60(71)$ \\
\hline \multicolumn{2}{|l|}{ Initial stage of breast cancer } \\
\hline Stage I-III & $67(80)$ \\
\hline Stage IV & $17(20)$ \\
\hline \multicolumn{2}{|l|}{ No. of previous endocrine therapy } \\
\hline 1 & $18(21.4)$ \\
\hline 2 & $43(51.2)$ \\
\hline 3 & $22(26.2)$ \\
\hline 4 & $1(1.2)$ \\
\hline Median (range) & $2(1-4)$ \\
\hline \multicolumn{2}{|l|}{ No. of previous chemotherapy } \\
\hline 0 & $39(46.3)$ \\
\hline 1 & $15(17.8)$ \\
\hline 2 & $11(13.1)$ \\
\hline 3 & $6(7 \cdot 1)$ \\
\hline 4 & $4(4.8)$ \\
\hline$\geq 5$ & $9(10.7)$ \\
\hline Median (range) & $2(0-11)$ \\
\hline \multicolumn{2}{|l|}{ Adjuvant endocrine treatment $(n=67)$} \\
\hline Yes & $53(79.1)$ \\
\hline No & $14(20.9)$ \\
\hline \multicolumn{2}{|l|}{ Disease extent } \\
\hline Visceral metastasis & $49(58.3)^{\mathrm{a}}$ \\
\hline Lung & 34 \\
\hline Liver & 16 \\
\hline Brain & 4 \\
\hline Bone marrow & 4 \\
\hline Adrenal gland & 4 \\
\hline Other (pancreas, kidney, ovary) & 5 \\
\hline Non-visceral metastasis & $35(41.7)$ \\
\hline Bone only & 17 \\
\hline Bone and nodes & 11 \\
\hline Soft tissue & 7 \\
\hline \multicolumn{2}{|l|}{ Tumor response to fulvestrant } \\
\hline Complete response & 0 \\
\hline Partial response & $13(15 \cdot 5)$ \\
\hline Stable disease & $21(25.0)$ \\
\hline Progressive disease & $50(59 \cdot 5)$ \\
\hline Disease control rate & $34(40.5)$ \\
\hline $95 \%$ Confidence interval & $30.5-51.5$ \\
\hline
\end{tabular}

Values are presented as median (range) or number (\%).

${ }^{a}$ Among them, 10 patients had multiple visceral involvement. ceptor (ER) down-regulator that competitively binds to ERs and induces a conformational change $[2,3]$. It does not show cross-resistance with tamoxifen or the ER agonist activity associated with tamoxifen [3]. The clinical efficacy of fulvestrant has been demonstrated in patients with ER-positive breast cancer that was previously untreated or treated with endocrine therapy [4-7].

Recently, several novel agents were developed to overcome resistance to endocrine therapies. Palbociclib is an orally bioavailable selective inhibitor of cyclin-dependent kinase 4 and 6 (CDK4 and CDK6) that prevents DNA synthesis by blocking progression of the cell cycle from $\mathrm{G} 1$ to $S$ phase $[8,9]$. On February 19, 2016, the U.S. Food and Drug Administration approved palbociclib for use in combination with fulvestrant for the treatment of women with ER-positive, human epidermal growth factor receptor 2 (HER2)-negative MBC with disease progression following endocrine therapy [10]. In the PALOMA-3 (Palbociclib Ongoing Trials in the Management of Breast Cancer 3) study, fulvestrant plus palbociclib was compared with fulvestrant plus placebo in patients with MBC that progressed on previous endocrine therapy; the median follow-up was 8.9 months. The median progression-free survival (PFS) was 9.5 months (95\% confidence interval [CI], 9.2 to 11.0) in the fulvestrant plus palbociclib group and 4.6 months (range, 3.5 to 5.6) in the fulvestrant plus placebo group (hazard ratio [HR], $0.46 ; 95 \% \mathrm{CI}, 0.36$ to $0.59 ; p<0.0001$ ) [11]. Fulvestrant thus became the preferred regimen partner of the $\mathrm{CDK}_{4}$ and CDK6 inhibitor palbociclib. In this context, here we focused on the specific clinical role of fulvestrant.

The results of a clinical trial comparing fulvestrant $250 \mathrm{mg}$ with anastrozole in postmenopausal women with MBC who showed disease progression after receiving endocrine treatment have been reported. The median time to progression (TTP) was 5.5 months in the fulvestrant arm and 4.1 months in the anastrozole arm; the overall response rate (ORR) was $19.2 \%$ and $16.5 \%$ for fulvestrant and anastrozole, respectively ( $95 \% \mathrm{CI}, 2.27$ to 9.05; $p=0.31$ ) [6]. The multicenter phase III Evaluation of Faslodex versus Exemestane Clinical Trial (EFECT) trial demonstrated similar efficacy of fulvestrant at $250 \mathrm{mg}$ compared with exemestane at $25 \mathrm{mg}$ orally once-daily. The median TTP was 3.7 months in both groups $(p=$ 0.653 ) and the ORR was $7.4 \%$ and $6.7 \%$ in the fulvestrant arm and the exemestane $\operatorname{arm}(p=0.736)$, respectively [7]. 


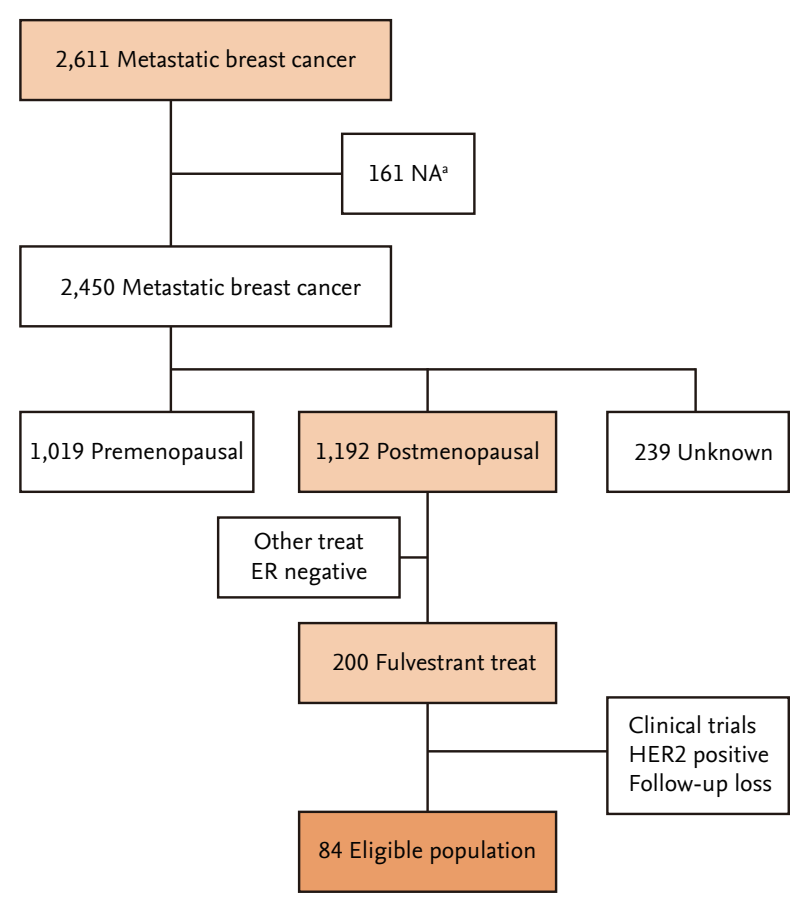

Figure 1. Flow diagram of patient inclusion in this study. NA, not available; ER, estrogen receptor; HER2, human epidermal growth factor receptor $2 .{ }^{\text {a }}$ Follow-up loss, double primary cancer, male.

However, few studies have reported data from Korean postmenopausal patients with MBC who were treated with fulvestrant. Moreover, objective data on fulvestrant are scarce. Knowledge of the clinical activity of fulvestrant would be informative to clinicians and helpful for patients. Here we report the clinical activity of fulvestrant for the treatment of postmenopausal women with hormone receptor-positive, HER2-negative MBC previously treated with endocrine therapy and/or chemotherapy.

\section{METHODS}

\section{Patients}

We retrospectively reviewed the medical records of all patients with MBC treated at Samsung Medical Center between January 2009 and August 2016. Eligible patients were postmenopausal women with MBC who had been treated with fulvestrant and whose tumors were ER-positive and/or progesterone receptor-positive. Patients with HER2-positive breast cancer and those who had received fulvestrant in clinical trials were excluded.
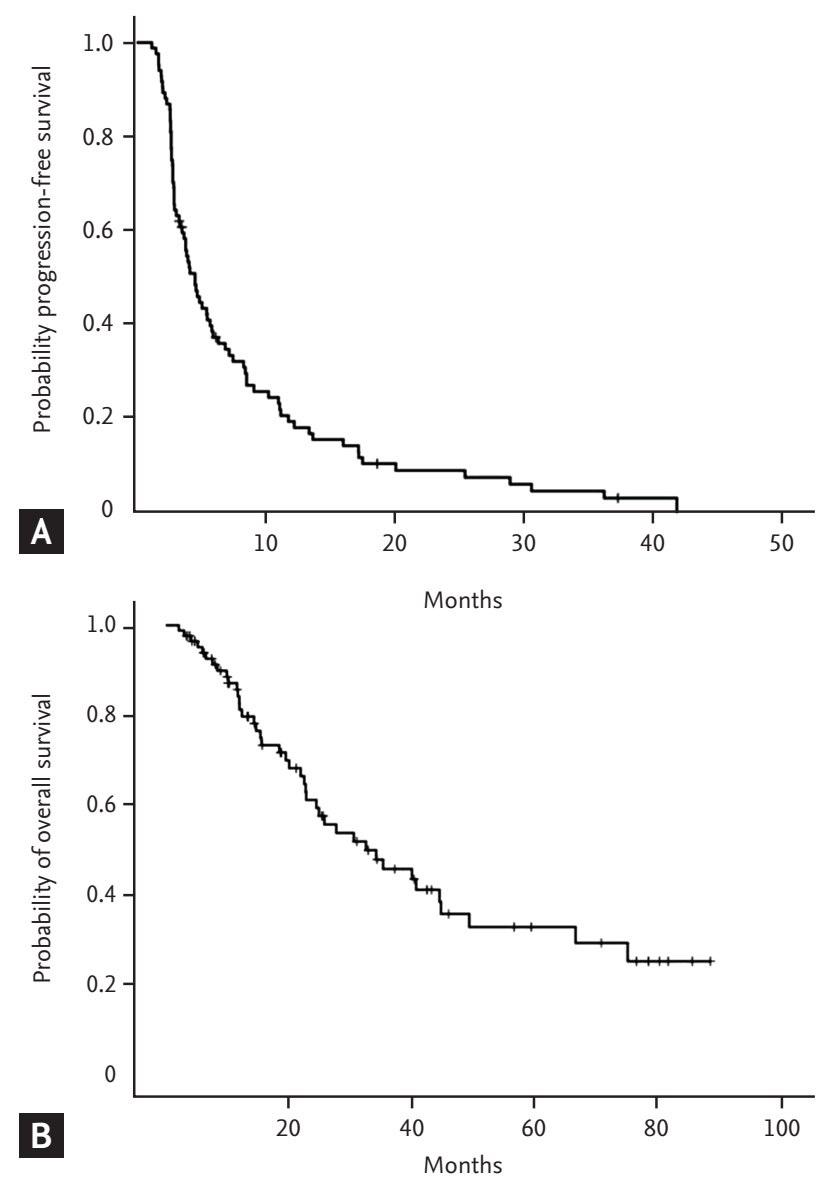

Figure 2. Clinical outcomes of fulvestrant in postmenopausal women previously treated with endocrine therapy (n = 84). (A) Progression-free survival (PFS) of patients treated with fulvestrant (median PFS, 4.4; 95\% confidence interval [CI], 3.4 to 5.5 months), (B) overall survival (OS) of patients treated with fulvestrant (median OS, $32.5 ; 95 \%$ CI, 17.6 to 47.4 months).

Patients received fulvestrant $250 \mathrm{mg}$ intramuscularly every 28 days (from January 2009 to November 2010) or $500 \mathrm{mg}$ intramuscularly every 28 days (from December 2010). We collected clinical data including baseline patient and tumor characteristics, prior endocrine and cytotoxic chemotherapies, and radiological findings. All procedures involving patients were reviewed and approved by the Institutional Review Board (IRB) of Samsung Medical Center, which waived informed consent (No. 2016-12-092).

\section{Response evaluation}

According to the relevant guidelines and our depart- 
Table 2. Characteristics of patients with tumor response $(n=13)$

\begin{tabular}{|c|c|c|c|c|c|c|c|c|c|c|}
\hline $\begin{array}{l}\text { Age, } \\
\text { yr }\end{array}$ & $\begin{array}{l}\text { Stage IV at } \\
\text { diagnosis }\end{array}$ & ER score & PgR score & $\begin{array}{c}\text { Adjuvant } \\
\text { HTx }\end{array}$ & $\begin{array}{l}\text { DFS, } \\
\text { mon }^{\mathrm{a}}\end{array}$ & HTx & $\begin{array}{l}\text { No. of } \\
\text { CTx }\end{array}$ & Lesion of metastasis & $\begin{array}{l}\text { PFS, } \\
\text { mon }\end{array}$ & $\begin{array}{l}\text { OS, } \\
\text { mon }\end{array}$ \\
\hline 77 & No & $3+5$ & $3+5$ & - & 303.0 & $\mathrm{~L}, \mathrm{~T}, \mathrm{E}$ & 3 & LN, bone, adrenal gland & $5 \cdot 3$ & 12.3 \\
\hline 58 & No & $2+5$ & 0 & A & 47.0 & $\mathrm{~A}, \mathrm{E}$ & 1 & LN, parasternal mass & 8.2 & 88.8 \\
\hline 62 & No & Unknown & Unknown & A & 26.8 & $\mathrm{~T}, \mathrm{E}$ & o & Pleural mass & 12.1 & 21.9 \\
\hline 65 & Yes & $2+5$ & $2+1$ & - & - & $\mathrm{L}, \mathrm{E}$ & 2 & Bone & $13 \cdot 3$ & $15 \cdot 5$ \\
\hline 71 & No & $2+5$ & $1+2$ & - & 101.1 & $\mathrm{~A}, \mathrm{E}$ & o & Bone & 15.9 & 78.7 \\
\hline 55 & Yes & $2+5$ & $2+3$ & - & - & $\mathrm{T}, \mathrm{L}, \mathrm{E}$ & 5 & LN, bone, lung, BM, brain & 17.1 & 40.4 \\
\hline 47 & No & $3+5$ & $2+2$ & $\mathrm{~T}$ & 28.3 & $\mathrm{~L}, \mathrm{E}$ & o & Bone & 17.1 & 76.8 \\
\hline 55 & No & $2+5$ & 0 & $\mathrm{~T}$ & 141.7 & $\mathrm{E}$ & 0 & LN, bone, lung & 20.0 & 43.2 \\
\hline 55 & No & $3+5$ & $2+1$ & $\mathrm{~T}+\mathrm{A}$ & 69.9 & $\mathrm{E}$ & $\mathrm{O}$ & $\mathrm{LN}$ & $25 \cdot 4$ & 80.5 \\
\hline 52 & No & $1+3$ & $2+5$ & $\mathrm{~L}$ & 28.6 & $\mathrm{~T}, \mathrm{E}$ & 0 & Lung & 30.5 & 56.8 \\
\hline 49 & No & Unknown & Unknown & $\mathrm{T}, \mathrm{L}$ & 169.0 & $\mathrm{E}$ & o & bone & 36.2 & 82.0 \\
\hline 59 & No & $3+5$ & $2+1$ & - & $55 \cdot 7$ & $\mathrm{~L}, \mathrm{E}$ & 4 & LN, lung & 37.2 & 37.2 \\
\hline 52 & No & $2+5$ & $2+5$ & $\mathrm{~T}$ & 24.0 & A & 0 & Liver & 41.8 & 71.0 \\
\hline
\end{tabular}

ER, estrogen receptor; PgR, progesteron receptor; HTx, endocrine therapy; DFS, disease free survival; CTx, systemic cytotoxic therapy; PFS, progression-free survival; OS, overall survival; L, letrozole; T, tamoxifen; E, exemestane; LN, lymph node; A, anastrozole; BM, bone marrow.

Time to metastatic breast cancer from operation.

ment policies, tumor response was assessed every 8 weeks ( \pm 1 week) and at the end of treatment, as well as when disease progression was suspected. Tumor responses were assessed by appropriate imaging techniques such as computed tomography, bone scans, and magnetic resonance imaging if indicated. The primary endpoint of this study was PFS; the secondary endpoints included overall survival (OS), disease control rate (DCR), and safety. The DCR included the rates of complete response, partial response (PR), and stable disease (SD). The responses were classified according to the Response Evaluation Criteria in Solid Tumors version 1.1 [12]. Adverse events were collected and graded according to the National Cancer Institute Common Terminology Criteria for Adverse Events version 4.03.

\section{Statistics}

Descriptive statistics are reported as proportions and medians. Kaplan-Meier estimates were used for the analysis of all time-to-event variables and the $95 \% \mathrm{CI}$ for the median time to event was computed. OS was measured from the date of fulvestrant treatment to the date of death from any cause and was censored at the date of the last follow-up visit. PFS was calculated from the date of fulvestrant treatment to the date of disease progression, death from any cause, or the last follow-up. The DCR is presented with a 95\% CI. Univariate and multivariate analyses examined the impact of clinical and treatment parameters on the survival outcome using Cox proportional hazards analyses. Variables used to identify prognostic parameters for PFS and OS were age, presence of visceral disease, stage at diagnosis, prior adjuvant endocrine therapy, prior cytotoxic therapy, time to surgery to recurrence, and time from first-line endocrine therapy to fulvestrant. Nonsignificant variables were dropped individually, beginning with the least significant variable. Variables with $p<0.05$ were considered significant for the analysis, and the $95 \%$ CIs were calculated. All statistical analyses were performed using PASW Statistics version 23.0 (SPSS Inc., Chicago, IL, USA).

\section{RESULTS}

\section{Patient characteristics}

Between January 2009 and August 2016, a total of 200 patients with $\mathrm{MBC}$ were treated with fulvestrant. Among them, 116 patients were excluded because they were tak- 
ing part in a clinical study, had HER2-positive MBC, or lacked available follow-up medical records. Thus, a total of 84 patients were finally included in this study. Fig. 1 shows a flow chart of patient inclusion.

The patient baseline characteristics are summarized in Table 1. The median patient age was 57.7 years (range, 31 to 82). All patients had been treated with at least one endocrine treatment before fulvestrant therapy, including tamoxifen, anastrozole, letrozole, or exemestane. A median of two previous endocrine treatments had been given; $79 \%$ of the patients had received two or more endocrine treatments. Forty-five patients (54\%) were treated with systemic chemotherapy for MBC, with a median of two chemotherapy regimens. All patients had cancer metastasis and visceral disease was present in 49 patients (58\%). Of the patients with visceral disease, 10 had multiple visceral organ involvement. The most common organ of visceral metastasis was the lung, which was present in 34 patients. The other visceral metastases were as follows: liver $(n=16)$, brain $(n=4)$, bone marrow $(n=4)$, adrenal gland $(n=4)$, and other organs $(n=5)$ such as pancreas, kidney, and ovary. Regarding non visceral disease $(n=35), 17$ patients had bone-only metastasis, 11 had both node and bone metastasis, and seven patients had soft tissue metastasis.

\section{Clinical outcomes and safety}

All analyzed patients received fulvestrant for a median treatment time of 3.2 months (range, 1 to 41 ). The median follow-up duration was 36.4 months (95\% CI, 25.2 to 47.6). The estimated median PFS and OS were 4.4 months (95\% CI, 3.4 to 5.5) and 32.5 months (95\% CI, 17.6 to 47.4 ), respectively (Fig. 2). The DCR was $40.5 \%$ (95\% CI, 30.5 to 51.5); PR was observed in $16 \%$ and SD in $25 \%$ of all patients. Among patients with $\mathrm{PR}(\mathrm{n}=13,16 \%)$, the response duration was 17.1 months ( $95 \% \mathrm{CI}, 12.4$ to 21.9 ). The details of these 13 patients are shown in Table 2.

The therapy-related adverse events are summarized in Table 3. The most frequently reported adverse reactions were mild-to-moderate grade myalgia (10.5\% of the patients), injection site pain ( $7 \%)$, and fatigue (7\%). Fulvestrant was generally well tolerated. Dose reduction was not required in any of the patients, and no patient discontinued fulvestrant due to adverse events. There were no therapy-related deaths.
Table 3. All grades of adverse events

\begin{tabular}{lc}
\hline Type of AE & No. $(\%)$ \\
\hline All patients with AEs & $36(41.9)$ \\
Myalgia, arthralgia & $9(10.5)$ \\
Injection site pain & $6(7.0)$ \\
Fatigue & $6(7.0)$ \\
Anorexia & $5(5.8)$ \\
Hot flush & $4(4.7)$ \\
Nausea & $2(2.3)$ \\
Pruritus & $2(2.3)$ \\
Infectiona & $2(2.3)$ \\
\hline
\end{tabular}

AE, adverse event.

${ }^{\text {a Pneumonia }}(n=1)$, herpes zoster $(n=1)$.

\section{DISCUSSION}

ER-positive breast cancer represents the most common subtype in both premenopausal and postmenopausal women. AIs and tamoxifen are currently the preferred first-line therapy for ER-positive postmenopausal women with MBC. In the second-line setting, tamoxifen and AIs are used sequentially. Several clinical trials of second-line therapy in metastatic or locally advanced breast cancer for postmenopausal women have shown similar efficacy of these therapies compared to other endocrine therapies, with good tolerability profiles $[6,7,13]$. The effectiveness of fulvestrant $250 \mathrm{mg}$ was determined by comparing the results for ORR and TTP to those of anastrozole $1 \mathrm{mg}$. The median TTP was 5.5 months in the fulvestrant arm and 4.1 months in the anastrozole arm (HR, 0.95; 95\% CI, 0.82 to $1.10 ; p=0.48$ ) and the ORR was $19.2 \%$ and $16.5 \%$ for fulvestrant and anastrozole, respectively (95\% CI, 2.27 to 9.05; $p=0.31$ ). There was no statistically significant difference in OS between the two treatment groups after a follow-up duration of 27 months [6,13]. In the EFECT trial, the effectiveness of fulvestrant $250 \mathrm{mg}$ was compared with that of exemestane. The median TTP was 3.7 months in both groups (HR, 0.96; 95\% CI, 0.82 to $1.13 ; p=0.653$ ), whereas the ORR was $7.4 \%$ and $6.7 \%$ in the fulvestrant arm and in the exemestane arm, respectively (HR, 1.12; 95\% CI, 0.58 to $2.19 ; p=0.736)[7]$.

The regulatory authorities of many countries have approved fulvestrant for use in MBC after progression on previous endocrine treatment. However, in South Korea, 
patients are not reimbursed for the use of fulvestrant for ER-positive breast cancer in any situation. We focused on the clinical activity of fulvestrant in postmenopausal women with ER-positive MBC who were treated with endocrine therapy and/or chemotherapy in real clinical practice. In our study the median patient age was 57.7 years (range, 31 to 82); the peak age range of breast cancer incidence in Korea is 45 to 49 years, followed by 50 to 59 years [14]. The DCR was $40.5 \%$ (95\% CI, 30.5 to 51.5 ), PR was observed in $16 \%$ of the patients, and SD was observed in $25 \%$ of the patients, with a mean response duration of 17.1 months (95\% CI, 12.4 to 21.9). The therapy-related adverse events were tolerable; however, the toxicity profiles might be underestimated due to the retrospective nature of this analysis. Our results are consistent with existing literature suggesting that fulvestrant is an effective second-line therapy for metastatic or locally advanced breast cancer in postmenopausal women. Moreover, approximately $79 \%$ of the patients in our study had undergone two or more endocrine treatments and 54\% had been treated with systemic cytotoxic therapy. This finding suggests that fulvestrant has therapeutic efficacy as a salvage treatment in postmenopausal women who have been heavily treated. Due to the high cost of fulvestrant, it is necessary to better define the subgroup of patients who truly benefit from fulvestrant treatment. We found that age, presence of visceral disease, stage at diagnosis, prior adjuvant endocrine therapy, prior cytotoxic therapy, time to surgery to recurrence, and time from first-line endocrine therapy to fulvestrant did not significantly influence clinical outcomes (Supplementary Tables 1 and 2).

Our study has some limitations, including its small sample size, its retrospective nature, and the heterogeneous treatment schedules. These limitations might have affected the findings of this study. In addition, the doses of fulvestrant were not consistent: before November 2010, 24 patients received a fulvestrant dose of 250 mg intramuscularly every 28 days because optimal dose of fulvestrant has not been identified. In our study, the dose of fulvestrant did not influence PFS or OS (PFS: 4.4 months for the $250 \mathrm{mg}$ dose vs. 4.0 months for the $500 \mathrm{mg}$ dose, $p=0.938$; OS: 34.7 months vs. 22.7 months, respectively, $p=0.566$ ). However, these findings should be interpreted with caution. In the final report of the CONFIRM (Comparison of Faslodex in Recurrent or
Metastatic Breast Cancer) trial, fulvestrant 500 mg was associated with a $19 \%$ reduction in risk of death and a 4.1-month difference in median OS compared with fulvestrant $250 \mathrm{mg}$ in patients with locally advanced or metastatic ER-positive breast cancer. Fulvestrant 500 mg was well tolerated, and no new safety concerns were identified [15]. Fulvestrant also showed encouraging clinical activity and favorable feasibility in postmenopausal women with MBC who had been treated with multiple endocrine therapies and/or cytotoxic chemotherapies.

\section{KEY MESSAGE}

1. Fulvestrant showed encouraging clinical activity and favorable feasibility in postmenopausal women with metastatic breast cancer who had been treated with multiple endocrine therapies and/or cytotoxic chemotherapies.

2. Fulvestrant was generally well tolerated in patients with heavily treated metastatic breast cancer.

\section{Conflict of interest}

No potential conflict of interest relevant to this article was reported.

\section{REFERENCES}

1. Cardoso F, Costa A, Norton L, et al. ESO-ESMO 2nd international consensus guidelines for advanced breast cancer (ABC2). Breast 2014;23:489-502.

2. Robertson JF, Erikstein B, Osborne KC, et al. Pharmacokinetic profile of intramuscular fulvestrant in advanced breast cancer. Clin Pharmacokinet 2004;43:529-538.

3. Wakeling AE, Dukes M, Bowler J. A potent specific pure antiestrogen with clinical potential. Cancer Res 1991;51:3867-3873.

4. Howell A, Robertson JF, Abram P, et al. Comparison of fulvestrant versus tamoxifen for the treatment of advanced breast cancer in postmenopausal women previously untreated with endocrine therapy: a multinational, double-blind, randomized trial. J Clin Oncol 2004;22:1605-1613.

5. Howell A, Robertson JF, Quaresma Albano J, et al. Fulves- 
trant, formerly ICI 182,780 , is as effective as anastrozole in postmenopausal women with advanced breast cancer progressing after prior endocrine treatment. J Clin Oncol 2002;20:3396-3403.

6. Robertson JF, Osborne CK, Howell A, et al. Fulvestrant versus anastrozole for the treatment of advanced breast carcinoma in postmenopausal women: a prospective combined analysis of two multicenter trials. Cancer 2003;98:229-238.

7. Chia S, Gradishar W, Mauriac L, et al. Double-blind, randomized placebo controlled trial of fulvestrant compared with exemestane after prior nonsteroidal aromatase inhibitor therapy in postmenopausal women with hormone receptor-positive, advanced breast cancer: results from EFECT. J Clin Oncol 2008;26:1664-1670.

8. Fry DW, Harvey PJ, Keller PR, et al. Specific inhibition of cyclin-dependent kinase 4/6 by PD 0332991 and associated antitumor activity in human tumor xenografts. Mol Cancer Ther 2004;3:1427-1438.

9. Toogood PL, Harvey PJ, Repine JT, et al. Discovery of a potent and selective inhibitor of cyclin-dependent kinase 4/6. J Med Chem 2005;48:2388-2406.

10. Walker AJ, Wedam S, Amiri-Kordestani L, et al. FDA approval of palbociclib in combination with fulves- trant for the treatment of hormone receptor-positive, HER2-negative metastatic breast cancer. Clin Cancer Res 2016;22:4968-4972.

11. Cristofanilli M, Turner NC, Bondarenko I, et al. Fulvestrant plus palbociclib versus fulvestrant plus placebo for treatment of hormone-receptor-positive, HER2-negative metastatic breast cancer that progressed on previous endocrine therapy (PALOMA-3): final analysis of the multicentre, double-blind, phase 3 randomised controlled trial. Lancet Oncol 2016;17:425-439.

12. Eisenhauer EA, Therasse P, Bogaerts J, et al. New response evaluation criteria in solid tumours: revised RECIST guideline (version 1.1). Eur J Cancer 2009;45:228-247.

13. Howell A, Pippen J, Elledge RM, et al. Fulvestrant versus anastrozole for the treatment of advanced breast carcinoma: a prospectively planned combined survival analysis of two multicenter trials. Cancer 2005;104:236-239.

14. Jung YS, Na KY, Kim KS, et al. Nation-wide Korean breast cancer data from 2008 using the breast cancer registration program. J Breast Cancer 2011;14:229-236.

15. Di Leo A, Jerusalem G, Petruzelka L, et al. Final overall survival: fulvestrant $500 \mathrm{mg}$ vs $250 \mathrm{mg}$ in the randomized CONFIRM trial. J Natl Cancer Inst 2014;106:djt337. 
Heo $\mathrm{MH}$, et al. Fulvestrant for salvage treatment

Supplementary Table 1. The prognostic factors that affected patients survival the development of metastasis in univariate survival analysis

\begin{tabular}{|c|c|c|c|c|}
\hline \multirow{2}{*}{ Characteristic } & \multicolumn{2}{|c|}{ Progression-free survival } & \multicolumn{2}{|c|}{ Overall survival } \\
\hline & Hazard ratio $(95 \% \mathrm{CI})$ & $p$ value & Hazard ratio $(95 \% \mathrm{CI})$ & $p$ value \\
\hline $\operatorname{Age}^{a}(\geq 57$ year $)$ & $1.065(0.680-1.670)$ & 0.782 & $1.242(0.666-2.315)$ & 0.495 \\
\hline Time to recurrence from surgery ( $<5$ years) & $1.049(0.639-1.723)$ & 0.850 & $1.131\left(0.575^{-2.228)}\right.$ & 0.721 \\
\hline $\begin{array}{l}\text { Time to fulvestrant from 1st endocrine } \\
\text { therapy ( }<5 \text { years) }\end{array}$ & $1.052(0.613-1.806)$ & 0.855 & $1.267(0.654-2.455)$ & 0.483 \\
\hline Stage at diagnosis (stage IV) & $1.453(0.830-2.541)$ & 0.191 & $2.387(1.065-5.351)$ & 0.035 \\
\hline Adjuvant endocrine therapy (received) & $0.990(0.625-1.569)$ & 0.966 & $0.599(0.321-1.117)$ & 0.107 \\
\hline Chemotherapy ( $\geq 2$ regimen) & $1.464(0.914-2.346)$ & 0.113 & $2.254(1.208-4.207)$ & 0.011 \\
\hline Visceral metastasis (present) & $1.152(0.732-1.813)$ & 0.540 & $1.498(0.793-2.832)$ & 0.213 \\
\hline
\end{tabular}

CI, confidence interval.

${ }^{\mathrm{a}}$ Age at time of fulvestrant therapy. 
Supplementary Table 2. Multivariate analysis of associations for clinical features with overall survival

\begin{tabular}{lcc}
\hline \multirow{2}{*}{ Characteristic } & \multicolumn{2}{c}{ Overall survival } \\
\cline { 2 - 3 } & Hazard ratio (95\% CI) & $p$ value \\
\hline Stage at diagnosis (stage IV) & $1.848(0.705-4.843)$ & 0.212 \\
Adjuvant endocrine therapy (received) & $0.889(0.412-1.918)$ & 0.765 \\
Chemotherapy ( $\geq 2$ regimen) & $2.017(0.967-3.712)$ & 0.071 \\
Visceral metastasis (present) & $1.413(0.736-2.715)$ & 0.299 \\
\hline
\end{tabular}

CI, confidence interval. 